independent screening efforts. In order to fill in the topography of the evolving cancer dependency map, more cancer cell lines with broader genetic diversity need to be analyzed. The potential for combining larger data sets will allow us to complete the dependency map sooner and hopefully unveil a treasure trove of new therapeutic cancer targets.

\section{References}

1. DepMap Achilles $19 Q 1$ Public. Available online at: https://figshare.com/ articles/DepMap_Achilles_19Q1_Public/7655150/1 (last accessed January 28, 2020).

2. Behan FM, lorio F, Picco G, et al. Prioritization of cancer therapeutic targets using CRISPR-Cas9 screens. Nature 2019;568:511-516. DOI: 10.1038/s41586-019-1103-9.

3. Hart T, Chandrashekhar M, Aregger M, et al. High-resolution CRISPR screens reveal fitness genes and genotype-specific cancer liabilities. Cell 2015;163:1515-1526. DOI: 10.1016/j.cell.2015.11.015.

4. Meyers RM, Bryan JG, McFarland JM, et al. Computational correction of copy number effect improves specificity of CRISPR-Cas9 essentiality screens in cancer cells. Nat Genet 2017;49:1779-1784. DOI: 10.1038/ng.3984.
5. Shi J, Wang E, Milazzo JP, et al. Discovery of cancer drug targets by CRISPR-Cas9 screening of protein domains. Nat Biotechnol 2015;33:661667. DOI: $10.1038 / \mathrm{nbt} .3235$.

6. Tzelepis K, Koike-Yusa H, De Braekeleer E, et al. A CRISPR dropout screen identifies genetic vulnerabilities and therapeutic targets in acute myeloid leukemia. Cell Rep 2016;17:1193-1205. DOI: 10.1016/j.celrep.2016.09.079.

7. Wang $\mathrm{T}, \mathrm{Yu} \mathrm{H}$, Hughes NW, et al. Gene essentiality profiling reveals gene networks and synthetic lethal interactions with oncogenic Ras. Cell 2017;168:890-903.e15. DOI: 10.1016/j.cell.2017.01.013.

8. DepMap at Broad Institute. Cancer dependency map. Available online at: https://depmap.org/portal/ (last accessed January 28, 2020).

9. DepMap at Sanger Institute. Available online at: https:// score.depmap.sanger.ac.uk/ (last accessed January 28, 2020).

10. Dempster JM, Pacini C, Pantel S, et al. Agreement between two large pancancer CRISPR-Cas9 gene dependency data sets. Nat Commun 2019;10:5817. DOI: 10.1038/s41467-019-13805-y.

11. Johnson WE, Li C, Rabinovic A. Adjusting batch effects in microarray expression data using empirical Bayes methods. Biostatistics 2007;8:118127. DOI: $10.1093 /$ biostatistics/kxj037.

12. Tsherniak A, Vazquez F, Montgomery PG, et al. Defining a cancer dependency map. Cell 2017;170:564-576.e16. DOI: 10.1016/ j.cell.2017.06.010.

\title{
A Jumbo Formation in the Viral Game Plan
}

\author{
Jeffrey K. Cornuault ${ }^{1,2}$ and Sylvain Moineau ${ }^{1,2,3, *}$
}

\section{Two reports describe the surprising "pan-CRISPR" resistance of jumbo phages against DNA-targeting CRISPR-Cas systems by erecting a nucleus-like structure during infection.}

More than a century after their discovery, bacteriophages (phages) are still full of unknowns. More scientists should be studying their biology, as they are a treasure trove of surprises! One source of astonishment is how they cope with the mechanisms used by bacteria to disable them. The seemingly endless arms race between phages and their hosts has led to the emergence of elaborate defense systems dedicated to the protection of the bacterial cell. ${ }^{1}$ In parallel, phages have mounted very successful and diverse countermeasures that have helped them to become the most abundant biological entities on the planet. ${ }^{2}$ These offensive and defensive schemes also became extraordinary sources of research tools in the biological sciences.

The relatively recent discovery and understanding of CRISPR-Cas systems has revealed the existence of an adaptive immune system in bacteria and archaea, which can efficiently defeat phages. As CRISPR Journal readers well know, this natural defense system generally works in three steps. First, the system incorporates shorts "spacers" from invading nucleic acids, such as phage genomes, into the CRISPR array. These spacers, which serve as memories of past invasions, are then transcribed and processed into short CRISPR RNAs (crRNAs) that will bind to Cas proteins. Finally, the crRNAs serve as guides for Cas endonucleases to bind and cut complementary sequences during subsequent invasion of viral genomes. $^{3}$

Viruses use various ways to escape CRISPR-Cas systems. For example, spontaneous mutations occurring during viral replication can prevent recognition of the targeted sequence. ${ }^{4}$ Another means to block the CRISPRCas system is to modify its nucleotides. ${ }^{5}$ Recently,

${ }^{1}$ Département de biochimie, de microbiologie et de bio-informatique, Faculté des sciences et de génie, Université Laval, Québec, Canada; ${ }^{2}$ Groupe de recherche en écologie buccale, Faculté de médecine dentaire, Université Laval, Québec, Canada; and ${ }^{3}$ Félix d'Hérelle Reference Center for Bacterial Viruses, Université Laval, Québec, Canada.

*Address correspondence to: Sylvain Moineau, PhD, Department of Biochemistry, Microbiology, and Bioinformatics, Faculty of Sciences \& Engineering, 1045 Avenue de la Médecine, Pavillon Vachon, Université Laval, Québec city, Quebec, Canada, G1V 0A6, Email: Sylvain.Moineau@bcm.ulaval.ca 
studies have shown the existence of families of phage proteins that directly inhibit CRISPR-Cas systems-the so-called anti-CRISPR proteins (ACR). ${ }^{6}$ Each of these ACR families is specialized in the inhibition of a particular CRISPR-Cas type and/or subtype, and several modes of action have been reported. ${ }^{7}$

By analyzing additional phages able to infect CRISPRimmune strains, two groups have recently reported an exciting new viral mechanism used to bypass CRISPRCas systems. ${ }^{8,9}$ Indeed, phages phiKZ and PCH45 can replicate on strains of Pseudomonas and Serratia, respectively, which, based on the CRISPR-Cas mode of action described above, they should not.

A pair of exciting reports from the labs of Joseph Bondy-Denomy and Peter Fineran solved the conundrum. It turns out that these bacterial viruses, nicknamed

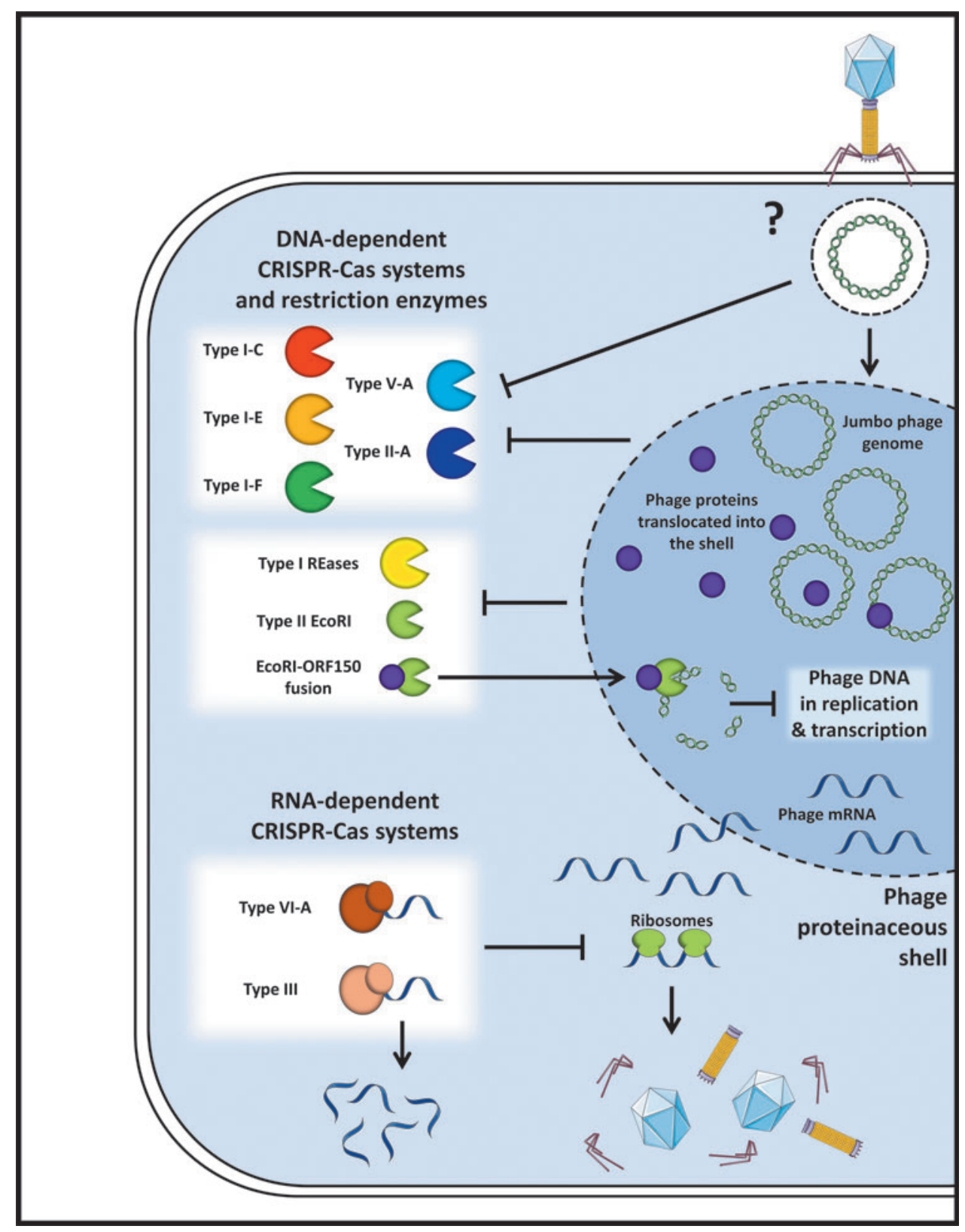

FIG. 1. Nucleus-like phage shell impacts bacterial defenses. A phage proteinaceous shell protects the jumbo phage double-stranded DNA genome from degradation by CRISPR-Cas systems and restriction enzymes. However, the shell does not protect phage mRNAs from RNA-targeting CRISPR-Cas systems (types III and VI-A), as they can reach the bacterial cytoplasm. (Images are reproduced from Smart Servier Medical Art.) 
"jumbo" because they have a large genome and capsid, are "resistant" to endogenous DNA-targeting CRISPRCas systems, such as subtypes I-C, I-E, and I-F, and to exogenous CRISPR-Cas systems from other bacterial species, such as type II-A from Streptococcus pyogenes or V-A from Moraxella bovoculi. Interestingly, these phages can also sidestep two types of restriction enzymes. The expression of HsdRM (R-M system type I) or EcoRI (type II) in Pseudomonas did not protect the cells against Jumbo phage infection. Bioinformatic analyses of their large genomes did not identify mutations, DNA modifications, or a known ACR, opening up the field to perhaps a new twist. And it did.

Three years ago, researchers showed that jumbo phages infecting Pseudomonas are able to form a proteinaceous shell structure inside a bacterial cell, reminiscent of a eukaryotic nucleus, where the phage replication and transcription take place. ${ }^{10,11}$ The shell is an assembly of one protein and is positioned mid-cell via the action of a tubulin-like protein, PhuZ. Homologous genes encoding proteins involved in shell assembly and localization reside in the genome of the jumbo phages used in these studies.

Using fluorescent microscopy ${ }^{9}$ and fluorescent confocal microscopy ${ }^{8}$ the Bondy-Denomy and Fineran groups demonstrated that key intracellular proteins of the CRISPRCas system (as well as the restriction enzymes) are blocked outside the phage shell, preventing access to the viral genome. In fact, this viral barrier is somewhat selective, as only proteins involved in phage DNA replication and transcription can reach inside the shell. Interestingly, fusion of the EcoRI restriction enzyme with a phage recombinase allows internalization of the endonuclease and specific cleavage of the jumbo phage's double-stranded genomic DNA (dsDNA). A similar strategy did not work with the large effector proteins/ complexes of CRISPR-Cas systems. These observations support the idea that the phage shell is able to filter proteins needed inside the shell and thwarts host defenses, thereby explaining the "pan-CRISPR" resistance of jumbo phages.

\section{Shell Game}

While most CRISPR-Cas studies have been on types that target DNA, a few systems can also target RNA. Both groups exploited this CRISPR-Cas diversity. They were able to block jumbo phage replication using bacterial strains carrying RNA-targeting CRISPR-Cas systems, namely a type III system in Serratia and type VI (cloned from Listeria) in Pseudomonas, suggesting that the bacterial immunity was achieved by targeting phage
mRNAs. It was also the first experimental demonstration that a type VI CRISPR-Cas system can target viral transcripts of a dsDNA phage to provide protection. In silico analyses of bacterial genomes to search for spacers matching parts of jumbo phage genomes revealed that they were found only in CRISPR arrays associated to a type III CRISPR-Cas system, supporting the experimental data.

The protective effect of the shell against bacterial defenses in two unrelated viral isolates suggests a widespread mechanism in jumbo phages. As CRISPR-Cas and restriction-modification systems are compatible inside a bacterial cell to improve overall phage resistance, ${ }^{12}$ the production of a shell is a crafty viral response to combat two prevalent host defenses. Yet, RNA-targeting CRISPR-Cas systems impeded the proliferation of these jumbo phages. It is tempting to speculate that some bacteria may have evolved their CRISPR-Cas systems to counteract such phage shells or perhaps they are byproducts of the defense arsenal against unknown RNA phages. As the nucleus-like structure appears to require one protein, one could envision smaller phages producing a similar protective device, but the production of a shell is also likely imposing a fitness cost, thereby affecting the rate of phage replication. This burden may not be suitable for most phages.

More generally, the presence of a replication compartment for DNA viruses to enhance their replication and protect their genomes has been known for several years in eukaryotic viruses. ${ }^{13}$ Moreover, a recent study highlighted the existence of an anti-phage system similar to existing ones in animal cells ${ }^{14}$ and adapted phage responses likely exists as well. The presence of numerous viral genes coding for phage proteins with unknown functions is still surprising to this day. If some of them are involved in counter defenses, more trick plays are surely waiting.

\section{References}

1. Labrie SJ, Samson JE, Moineau S. Bacteriophage resistance mechanisms. Nat Rev Microbiol 2010;8:317-327. DOI: 10.1038/nrmicro2315.

2. Samson JE, Magadán AH, Sabri M, et al. Revenge of the phages: defeating bacterial defences. Nat Rev Microbiol 2013;11:675-687. DOI: 10.1038/ nrmicro3096.

3. Makarova KS, Wolf $\mathrm{Yl}$, Iranzo J, et al. Evolutionary classification of CRISPR-Cas systems: a burst of class 2 and derived variants. Nat Rev Microbiol 2020;18:67-83. DOI: 10.1038/s41579-0190299-x.

4. Deveau H, Barrangou R, Garneau JE, et al. Phage response to CRISPRencoded resistance in Streptococcus thermophilus. J Bacteriol 2008;190:1390-1400. DOI: 10.1128/JB.01412-07.

5. Vlot M, Houkes J, Lochs SJA, Swarts DC, et al. Bacteriophage DNA glucosylation impairs target DNA binding by type I and II but not by type V CRISPR-Cas effector complexes. Nucleic Acids Res 2018;46: 873-885. DOI: 10.1093/nar/gkx1264. 
6. Bondy-Denomy J, Pawluk A, Maxwell KL, et al. Bacteriophage genes that inactivate the CRISPR/Cas bacterial immune system. Nature 2013;493:429-432. DOI: 10.1038/nature11723.

7. Hwang S, Maxwell KL. Meet the anti-CRISPRs: widespread protein inhibitors of CRISPR-Cas systems. CRISPR J 2019;2:23-30. DOI: 10.1089/ crispr.2018.0052.

8. Malone LM, Warring SL, Jackson SA, et al. A jumbo phage that forms a nucleus-like structure evades CRISPR-Cas DNA targeting but is vulnerable to type III RNA-based immunity. Nat Microbiol 2020;5:48-55. DOI: 10.1038/s41564-019-0612-5.

9. Mendoza SD, Nieweglowska ES, Govindarajan S, et al. A bacteriophage nucleus-like compartment shields DNA from CRISPR nucleases. Nature 2019;577:244-248. DOI: 10.1038/s41586-019-1786-y.

10. Chaikeeratisak V, Nguyen K, Egan ME, et al. The phage nucleus and tubulin spindle are conserved among large Pseudomonas phages. Cell Rep 2017;20:1563-1571. DOI: 10.1016/j.celrep.2017 .07.064.

11. Chaikeeratisak V, Nguyen K, Khanna K, et al. Assembly of a nucleus-like structure during viral replication in bacteria. Science 2017;355:194-197. DOI: $10.1126 /$ science.aal2130.

12. Dupuis $M E$, Villion $M$, Magadán AH, et al. CRISPR-Cas and restrictionmodification systems are compatible and increase phage resistance. Nat Commun 2013;4:2087-2087. DOI: 10.1038/ncomms 3087.

13. Schmid M, Speiseder T, Dobner T, et al. DNA virus replication compartments. J Virol 2014;88:1404-1420. DOI: 10.1128/jvi .02046-13.

14. Cohen D, Melamed S, Millman A, et al. Cyclic GMP-AMP signalling protects bacteria against viral infection. Nature 2019;574:691-695. DOI: 10.1038/s41586-019-1605-5 